\title{
Hypersensitivity reactions to enoxaparin and unfractionated heparin in a pregnant woman with gestational thrombocytopenia: A case report
}

\author{
Fahad A S Al-Eidan ${ }^{1,2 *}$, Nourah Al-Qhtani ${ }^{2}$, Areej F Alsubaie ${ }^{2}$ and Badria M Al-Nouh ${ }^{1,2}$ \\ ${ }^{1}$ King Saud bin Abdulaziz University for Health Sciences, Riyadh, Saudi Arabia \\ ${ }^{2}$ King Abdulaziz Medical City, Riyadh, Saudi Arabia
}

\begin{abstract}
Background: The risk of venous thromboembolism (VTE) and arterial thrombosis (stroke and heart attack) increases in women during pregnancy. Enoxaparin (a low molecular weight heparin) and unfractionated heparin are extensively used in the VTE prophylaxis and therapeutic doses.

Aims: We retrospectively describe an apparent relationship between enoxaparin and unfractionated heparin (UFH) in a woman with gestational thrombocytopenia developing a hypersensitivity reactions.

Case presentation: we report a clinical scenario of a 43 years old pregnant women (gravida 18, para 12 and living 6) with co-morbid obesity (BMI 47.9) and well known to have a gestational thrombocytopenia during the last two pregnancies. She was administered enoxaparin $0.4 \mathrm{ml}$ ( $40 \mathrm{IU}$ ) daily for VTE prophylaxis. After three days, the patient came with hypersensitivity reactions like erythema, pain, swelling, irritation at the site of injection and rash all over the body (lower limbs, abdomen and neck) appeared after the first dose of enoxaparin. Hypersensitivity skin reaction to enoxaparin was suspected and immediately drug was withdrawn. The performed UFH skin test was negative, and then she was prescribed UFH 5000 units subcutaneous every 12 hours. The patient had similar reaction but a severe form and UFH was discontinued. Fondaparinux was the only nonheparin anticoagulant available and was prescribed for this patient.
\end{abstract}

Conclusions: The patient had an allergic drug reaction and provides an evidence of cross-allergenicity between enoxaparin and UFH. Nonheparin anticoagulant improves both rashes and gestational thrombocytopenia.

Abbreviations: ADR: adverse drug reaction; NOACs: new oral anticoagulants; VTE: venous thromboembolism; UFH: unfractionated heparin.

\section{Introduction}

The risks of venous thromboembolism (VTE) and arterial thrombosis (stroke and heart attack) are increased in pregnant women. Compared to non-pregnant woman, the risk of arterial thrombosis and VTE during pregnancy is increased 3-5 folds. Moreover, the risk postpartum is even more and increased up-to 20-fold [1].

Unfractionated heparin (UFH) and low molecular weight heparin (LMWH) are widely used for the prevention of VTE and therapeutic purposes. UFH is heterogenous mixture of glycosaminoglycans that bind to antithrombin via a unique pentasaccharide sequence and catalyse activation of thrombin, factor $\mathrm{Xa}$ and other clotting enzymes. LMWH are a group of anticoagulants derived from UFH by chemical or enzymatic depolymerisation methods. They consist of one third the molecular weight of UFH with a mean of 4000 to 5000 Kilo Dalton (KDa) $[2,3]$. Nowadays, LMWH replace UFH due to a greater inhibitory activity against factor $\mathrm{Xa}$, improved pharmacokinetic properties, longer half-life, and are associated with a significant lower risk of heparin-induced thrombocytopenia [4]. Adverse drug reactions due to administration of heparins include immunologically mediated hypersensitivity reactions: heparin-induced thrombocytopenia, immediate hypersensitivity reactions and delayed hypersensitivity skin reactions such as large, symptomatic, erythematous infiltrated plaques at injection sites [5-7].

When hypersensitivity to LMWHs is confirmed using an adequate sensitive and specific available such as skin tests, standardized guidelines for selecting safe alternative nonheparin anticoagulant is still lacking [8]. Cross-reactivity among heparin including LMWHs has been reported in the literature around $37 \%(6,8)$. Proposed alternative nonheparin anticoagulants are heparinoids danaproid and pentosan polysulfate [2,5]. Fondaparinux is a synthetic pentasaccharide specifically bind to antithrombin in reversible manner and reinforces its inhibitory properties mainly towards factor Xa which is inhibited 300 times faster in the presence of fondaparinux. Despite the limited experience during pregnancy to a small cohort of patients, fondaparinux is actually considered to nonheparin alternative of choice in cases of hypersensitivity to UFH and LMWHs $[9,10]$. We

Correspondence to: Fahad A S Al-Eidan, Associate Professor, College of Medicine, King Saud bin Abdulaziz University for Health Sciences, Riyadh, Kingdom of Saudi Arabia, Tel: +966118011111 ext: 12234; Mobile: +966506414425; E-mail: faleidan@gmail.com, aidanf@ngha.med.sa

Key words: enoxaparin, fondaparinux, gestational thrombocytopenia, hypersensitivity, unfractionated heparin

Received: December 12, 2016; Accepted: December 26, 2016; Published: December 29, 2016 
retrospectively describe an apparent relationship between enoxaparin and unfractionated heparin (UFH) in a woman with gestational thrombocytopenia developing a hypersensitivity reactions.

\section{Method/Case presentation}

We report a clinical scenario of a 43 years old pregnant women (gravida 18, para 12 and living 6) with co-morbid obesity (BMI 47.9) and well known to have a gestational thrombocytopenia during the last two pregnancies. She was administered enoxaparin $0.4 \mathrm{ml}$ (40 IU) daily for VTE prophylaxis in the first week of second trimester. After three days, the patient came with hypersensitivity reactions like erythema, pain, swelling, irritation at the site of injection and rash all over the body (lower limbs, abdomen and neck) appeared on first time after the administration of enoxaparin. Hypersensitivity skin reaction to enoxaparin was suspected and immediately drug was withdrawn and the severity of signs and symptoms was substantially subsided. The performed UFH skin test was negative, and then subsequently she was prescribed UFH 5000 units subcutaneous every 12 hours. The patient had similar reaction with higher intensity and severe form and UFH was discontinued. Fondaparinux was the only nonheparin anticoagulant available and was prescribed for this patient and was continued over the gestational period. Patient was admitted to the hospital at term and fondaparinux was held 24 hours before caesarean section delivery and was resumed 12 hours after delivery throughout the post-partum period. There were no bleeding tendencies or VTE during fondaparinux therapy. Both baby and mother were well and discharged home. Oral informed consent were obtained since this case report does not involved personal images or data.

Hypersensitivity reaction was analysed and causality assessment performed using standard scales such as the WHO-UMC assessment scale, Naranjo's assessment scale and Karch-Lasagna assessment scale. Causality assessment was performed to link the time relationship between the hypersensitivity reaction and the suspected drug. The results of different assessment scales are reported in Table 1. Furthermore, other parameters were analysed to determine the severity, preventability, and predictability of the suspected hypersensitivity reaction and the result are shown in Table 2.

\section{Discussion}

Patient who receive heparin therapy (UFH or LMWH) may experience clinical complication such as hypersensitivity reactions in the skin. In our case, we describe a definite hypersensitivity reactions developed like erythema, pain, swelling, irritation at the site of injection and rash all over the body (lower limbs, abdomen and neck) after enoxaparin was administered and subsided after it was stopped. The patient had more severe symptoms when UFH administered after discontinuation of enoxaparin and there were no alternative causes for

Table 1. Causality assessment of the suspected hypersensitivity.

\begin{tabular}{|l|c|c|c|}
\hline ADR scale & WHO-UMC & Naranjo's & KARCH\&LASAGNA \\
\hline Assessment result & Certain & Definite & Definite \\
\hline
\end{tabular}

Table 2. Severity, preventability and predictability analyses of observed Hypersensitivity. the hypersensitivity reactions in both occasions.

Hypersensitivity to subcutaneous UFH and LMWH are induced by certain risk factors such as female gender, obesity and pregnancy which may contributed to hormonal or metabolic changes [11], which associated with our case report.

New oral anticoagulants (NOACs) have not yet been formally evaluated during pregnancy. LMWH and UFH are the safest option anticoagulants during pregnancy, however their use associated with increased immunological adverse reactions especially in obese pregnant women. Therefore, based on the present case report and its efficacy and tolerability, fondaparinux was the only non-heparin alternative anticoagulant option during pregnancy $[9,10]$. Safety of the foetus is always an issue when drug is prescribed for pregnant women. Therefore, prescribing fondaparinux for pregnant women should be limited to those patients with either severe hypersensitivity reaction to UFH and LMWH, as it happened in this case, or in the presence of immunological heparin-induced thrombocytopenia. Furthermore, gestational thrombocytopenia was normalised when fondaparinux was commenced.

\section{Conclusions}

The patient had an allergic drug reaction and provides an evidence of cross-allergenicity between enoxaparin and UFH. Fondaparinux a non-heparin anticoagulant improves both rashes and gestational thrombocytopenia.

\section{Ethics approval and Consent to participate}

Approved and given permission for publication and attached the memorandum.

\section{Consent for publication}

Obtained and available upon request.

\section{Availability of data and material}

Available on request.

\section{Competing interest}

Not applicable

\section{Funding}

No funding for this case report

\section{Authors' contribution}

F.A.S. Al-Eidan initiated the case report, analyzed and drafted the manuscript. Areej F Alsubaie, Badria M Al-Nouh contributed to the collection and interpretation of the data and revised the manuscript. All authors approved the final version of the manuscript.

\section{Disclosure of conflict of interest}

All authors have no conflict of interest to report.

\begin{tabular}{|l|l|}
\hline Assessment scale & Result \\
\hline Hartwig's Severity Scale & $\begin{array}{l}\text { Moderate Level 3 } \\
\text { (The ADR required that treatment with the suspected drug be held, discontinued, or otherwise changed, AND/OR an Antidote or other } \\
\text { treatment was required. No increase in length of stay) }\end{array}$ \\
\hline Preventability & Not Preventable \\
\hline Predictability & Unpredictable \\
\hline
\end{tabular}




\section{Acknowledgements}

None

\section{References}

1. Heit JA, Kobbervig CE, James AH, Petterson TM, Bailey KR, et al. (2005) Trends in the incidence of venous thromboembolism during pregnancy or postpartum: a 30-year population-based study. Ann Intern Med 143: 697-706.[Crossref]

2. Al-Eidan FA (2015) Pharmacotherapy of heparin-induced thrombocytopenia: therapeutic options and challenges in the clinical practices. $J$ Vasc Nurs 33: 10-20

3. Turpie AG (2004) Fondaparinux: a Factor Xa inhibitor for antithrombotic therapy. Expert Opin Pharmacother 5: 1373-1384.[Crossref]

4. Al-Eidan FA (2015) Is the incidence trend of heparin-induced thrombocytopenia decreased by the increased use of low-molecular-weight-heparin.Mediterr J Hematol Infect Dis 7: e2015029.

5. Trautmann A, Seitz CS (2009) Heparin allergy: delayed-type non-IgE-mediated allergic hypersensitivity to subcutaneous heparin injection. Immunol Allergy Clin North Am 3: 469-480.
6. Schindewolf M, Gobst C, Kroll H, Recke A, Louwen F, et al. (2013) High incidence of heparin-induced allergic delayed-type hypersensitivity reactions in pregnancy. $J$ Allergy Clin Immunol 132: 131-139.[Crossref]

7. Linkins LA, Dans AL, Moores LK (2012) Treatment and prevention of heparin-induced thrombocytopenia: Antithrombotic Therapy and Prevention of Thrombosis.(9th Edn) American College of Chest Physicians Evidence-Based Clinical Practice Guidelines. Chest 141(Suppl): e495S-e530S.

8. Bircher AJ, Harr T, Hohenstein L, Tsakiris DA (2006) Hypersensitivity reactions to anticoagulant drugs: diagnosis and management options. Allergy 61: 1432-1440. [Crossref]

9. Trog LM, Kahle B, Schindewolf M, Jappe U, Ludwig RJ (2015) Tolerance of Fondaparinux in Immediate-type Hypersensitivity to Heparins. Am J Med 128: e21-22. [Crossref]

10. Mazzolai L, Hohlfeld P, Spertini F, Hayoz D, Schapira M, et al. (2006) Fondaparinux is a safe alternative in case of heparin intolerance during pregnancy. Blood 108: 15691570.[Crossref]

11. Mendez J, Sanchis ME, de la Fuente R, Stolle R, Vega JM, et al. (1998) Delayed-type hypersensitivity to subcutaneous enoxaparin. Allergy 53: 999-100.

Copyright: $@ 2016$ Al-Eidan FAS. This is an open-access article distributed under the terms of the Creative Commons Attribution License, which permits unrestricted use, distribution, and reproduction in any medium, provided the original author and source are credited. 